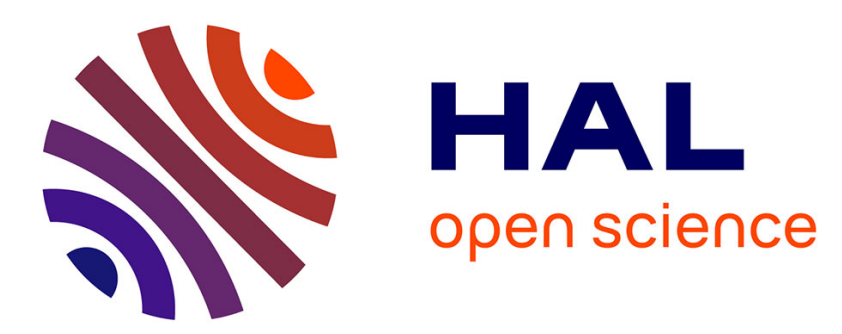

\title{
Shear Modification of long-chain branched polymers: a theoretical approach using POM-POM model
}

\author{
S. Bourrigault, Gérard Marin, Arnaud Poitou
}

\section{To cite this version:}

S. Bourrigault, Gérard Marin, Arnaud Poitou. Shear Modification of long-chain branched polymers: a theoretical approach using POM-POM model. Macromolecules, 2003, 36 (4), pp.1388 1394. 10.1021/ma021195t . hal-01007285

\section{HAL Id: hal-01007285 \\ https://hal.science/hal-01007285}

Submitted on 8 Feb 2017

HAL is a multi-disciplinary open access archive for the deposit and dissemination of scientific research documents, whether they are published or not. The documents may come from teaching and research institutions in France or abroad, or from public or private research centers.
L'archive ouverte pluridisciplinaire HAL, est destinée au dépôt et à la diffusion de documents scientifiques de niveau recherche, publiés ou non, émanant des établissements d'enseignement et de recherche français ou étrangers, des laboratoires publics ou privés. 


\title{
Shear Modification of Long-Chain Branched Polymers: A Theoretical Approach Using the Pom-Pom Model
}

\author{
S. Bourrigaud, ${ }^{\dagger}$ G. Marin, ${ }^{*, \dagger}$ and A. Poitou \\ LPCP, Université de Pau et des Pays de l'Adour, C.N.R.S. Avenue de l'U niversité, \\ BP1155 64013 Pau Cedex, France, and LMT-Cachan, Ecole Normale Supérieure de Cachan, \\ Université Paris 6, C.N.R.S. 61 Avenue du Président Wilson, 94235 Cachan Cedex, France
}

\begin{abstract}
"Shear modification" is a strong modification of rheological properties which affects mainly long-chain branched polymers like LDPE. The aim of this work is to explain this effect using recent advances in molecular dynamics and especially the pom-pom model which was designed for branched polymers. The original model was slightly modified in order to take into account the change in molecular topology related to the branch point withdrawal mechanism without introducing any further assumptions. A theoretical approach shows that when arm retraction is significant, the whole relaxation time scale is modified inducing a very different rheological behavior in shear and in elongation. Results are in qualitative agreement with reported experimental results, but a quantitative description requires some refinement in the molecular dynamics theory and would necessitate the use of well calibrated pom-pomshaped polymers for quantitative experimental evidence.
\end{abstract}

\section{Introduction}

When working on extrusion coating, extrusion casting, or film blowing processes, one meets difficulties to extrapolate results from a laboratory scale to semiindustrial and industrial scales. One explanation of those difficulties is the great differences in thermomechanical histories that the melt undergoes. Mainly branched polymers like LDPE are affected by a very important change in there rheological behavior. ${ }^{1,7}$ This well-known effect called "shear modification" or "shear refining" has been studied for a long time on an experimental point of view. ${ }^{1,2}$ This effect is usually neglected but is in fact of main importance. Actually, one who deals with processing needs to know which are the effective rheological properties of the material to extrapolate its behavior under process. On the other hand, a good understanding of this effect can be a very powerful tool to adjust material properties to the process in order to increase process and product performances. ${ }^{15}$

Shear modification is described to be a highly significant modification of rheological properties in both linear and nonlinear viscoelasticity domain and concerns mainly melts of long-chain branched molecules and high polydispersity. ${ }^{10,14,15}$ The most significant modifications are observed on the storage modulus ( $\left.G^{\prime}\right)$ in linear viscoelasticity at low frequency (decreasing of about $20 \%$ in some extreme cases), on the recoverable shear strain, ${ }^{6}$ transient shear, and normal force in nonlinear viscoel asticity, 6,14 and in the strain hardening behavior in uniaxial el ongation. ${ }^{15,16}$ L oss of die swell had been also reported extensively. ${ }^{1-5,10,11,14,17}$ Viscous properties undergo a small decrease, whenever the melt flow index is reported to be significantly increased. All those considerations gave authors arguments to conclude on a decrease in melt elasticity and strain hardening behavior.

The molecular origin of this phenomenon has been believed to be a disentanglement mechanism of long-

\footnotetext{
† Université de Pau et des Pays de l'Adour.

¥ Ecole Normale Supérieure de Cachan.

* Corresponding author: e-mail gerard.marin@univ-pau.fr.
}

chain branched molecules for a long time, ${ }^{1,2,9}$ but this idea was refuted by Leblans and Bastiaansen, ${ }^{15}$ considering the annealing time value. As the modification is fully reversible, one can define a time the modified melt needs to recover its initial rheological properties. For these authors this time was measured to be of the order of the maximum relaxation time which is a piece of evidence that deepest levels of side chains and molecular backbone are involved in the shear modification dynamics. A second piece of evidence lays in the analysis of solution treatment data. When samples are dissolved and repreci pitated in order to produce molecular disentanglement, rheological properties modifications are quite similar to those caused by shear modification; however, the corresponding annealing time is lower by 2 orders of magnitude.

As rheological properties of a processed melt are similar to those of linear polymers in el ongation (without any strain hardening behavior), a new mechanism was proposed: Iong side chain branches align each other along the backbone in a single tube. According to chain reptation theories of branched polymers, the longest time is associated with the backbone dynamics, which reptates since side chain branches have relaxed., $8,12,20$ The side chain branches alignment is therefore in contradiction with reptation theory because the shear modification lifetime is associated with a time of the order of the backbone relaxation time. The molecular explanation of shear modification is still today an open issue. The aim of this work is to discuss the side chain branches alignment hypothesis in the light of recent molecular dynamics picture on long-chain branched polymers using the pom-pom polymer model.

\section{Theory}

2.1. Pom-Pom Model. The pom-pom model was developed by MCLeish and Larson ${ }^{18}$ to describe the rheological behavior of melts with long-chain branching and appears to be a generalization of the $\mathrm{H}$-polymer structure. The molecules are composed of two "poms" considered as stars of $q$ arms of dimensionless length 
$S_{a}=M_{a} / M_{e}$ connected by a backbone of dimensionless length $S_{b}=M_{b} / M_{e}$, where $M_{a}, M_{b}$ and $M_{e}$ are respectively the molecular weights of arms, backbone, and entanglements. The main idea of this model is to consider three different hierarchical relaxation mechanisms. In a first step molecular orientation of the crossbar segment is consi dered. In a second step, as the branch points cause frictional drag force, McLeish and Larson introduced a backbone stretching parameter $\lambda$ with entropic elastic force of the stretched segment balanced by the frictional drag force of branch points. This mechanism occurs until the limiting stretching value of $q$ is reached which is determined by Gaussian chain statistics and equilibrium considerations. At last, branch point withdrawal is considered to occur when the flow is strong enough to induce backbone maximum stretching value of $q . s_{c}$ is then introduced as the $M_{d}$ $M_{e}$ ratio with $M_{c}$ the molecular weight of the withdrawn arm. This last mechanism is taken into account by adding an extra contribution to the stress tensor and by considering that the dangling arms length is reduced. The authors of the pom-pom model do not take into account the increase in tube length, and therefore the entanglement network remains basically controlled by the initial backbone volume fraction. They argue that the star-arm relaxation remains much faster on the time scale of their restricted flow conditions. We must notice here that the major effect of branch point withdrawal is to induce a decrease of the relaxation time scale.

We consider in our approach that, when branch point withdrawal occurs, the relaxation times are modified and have to account for both the reduction of the arm length and the increase of the backbone length. As was shown by McLeish and Larson, the local deformation rate within a tube segment surrounding a withdrawn arm segment is much larger than the bulk deformation rate. Therefore, even under the restricted flow conditions of these authors, the withdrawn arm fraction acts as a topological obstacle. This also means that the tube edges act as new branch points. We postulate therefore that under strong flow conditions the branch point withdrawal mechanism is very significant and induces some important changes in the molecular entanglement network so that the effect of chain retraction is to induce some significant changes, both increase and decrease, in the relaxation time spectrum.

2.1.1. Pom-Pom Arms Dynamics. In the original pom-pom model, the relaxation time of a segment of length $s_{a}-s_{c}$ is

$\tau_{\mathrm{a}}\left(\mathrm{s}_{\mathrm{c}}\right)=\tau \exp \left[\frac{15}{4} \mathrm{~S}_{\mathrm{a}}\left(\frac{\left(1-\mathrm{s}_{\mathrm{c}} \mathrm{s}_{\mathrm{a}}\right)^{2}}{2}-\left(1-\phi_{\mathrm{b}}\right) \frac{\left(1-\mathrm{s}_{\mathrm{c}} / \mathrm{s}_{\mathrm{a}}\right)^{3}}{3}\right)\right]$

where $\phi_{\mathrm{b}}$ the fraction of backbone material. Ball and McLeish ${ }^{13}$ defined the characteristic time $\tau$ as

$$
\tau=\tau_{0} \mathrm{~S}_{\mathrm{a}}^{2} \sqrt{\frac{\pi}{v\left(\mathrm{~S}_{\mathrm{a}}\right)}}
$$

where $\tau_{0}$ is the Rouse time and $v$ is a structural parameter relative to the chemical nature of the material, and its value was experimentaly measured to be 0.6 for polyethylene. ${ }^{19}$ The exponential is derived from the breathing modes of stars, and the subtraction inside the exponential is derived from the dynamic dilution effect which has a strong accelerating effect on the time scale of arms relaxation. ( $\left.1-\phi_{\mathrm{b}}\right)$ is the vol ume fraction of material involved in relaxing arms. In the case of flows considered by MCLeish and Larson, ${ }^{18}$ branch point withdrawal has a minor effect and can be reasonably neglected as was argued by these authors. N evertheless, in the case of stronger flows, a significant fraction of arm material is withdrawn into the backbone tube. To derive consistently our model from the pom-pom model, we have to take into account the reduction of arm length. Following this molecular picture, the definition of the characteristic time $\tau$ for the arm relaxation becomes

$$
\tau\left(\mathrm{s}_{\mathrm{c}}\right)=\tau_{0}\left(\mathrm{~S}_{\mathrm{a}}-\mathrm{s}_{\mathrm{c}}\right)^{2} \sqrt{\frac{\pi}{v\left(\mathrm{~S}_{\mathrm{a}}-\mathrm{S}_{\mathrm{c}}\right)}}
$$

Furthermore, the fraction of arm material withdrawn into the backbone tube does not contribute to the volume fraction of relaxing arms involved in the dynamic dilution effect. Therefore, when branch point withdrawal occurs, the effective volume fraction of relaxing arms becomes $1-\phi_{\mathrm{b}}-\phi_{\mathrm{c}}$, where $\phi_{\mathrm{c}}$ is the volume fraction of withdrawn material. We take into account this effective volume fraction of material outside the backbone tube by modifying expression 1 into

$$
\begin{aligned}
\tau_{\mathrm{a}}\left(\mathrm{S}_{\mathrm{c}}\right)=\tau\left(\mathrm{S}_{\mathrm{c}}\right) \exp \left[\frac { 1 5 } { 4 } \mathrm { S } _ { \mathrm { a } } \left(\frac{\left(1-\frac{\mathrm{S}_{\mathrm{c}}}{\mathrm{S}_{\mathrm{a}}}\right)^{2}}{2}-\right.\right. & \\
& \left.\left.\left(1-\phi_{\mathrm{b}}-\phi_{\mathrm{c}}\right) \frac{\left.\left.\left(1-\frac{\mathrm{S}_{\mathrm{c}}}{\mathrm{S}_{\mathrm{a}}}\right)^{3}\right)\right]}{3}\right)\right]
\end{aligned}
$$

where

$$
\phi_{c}=\frac{2 q s_{c}}{2 q s_{a}+s_{b}}
$$

We can notice here that taking into account the material outside the tube is a small conceptual difference from the original pom-pom model which can have however a significant effect on the relaxation time scale. Nevertheless, $s_{c}$ must be of the order of $s_{a}$ so that the fraction of withdrawn material $\phi_{c}$ cannot be neglected $\left(0.1<\phi_{\mathrm{c}}<1\right)$, which means that shear modification occurs only in strong flows situations (high strain rates and high strains). As we can see, if $\mathrm{s}_{\mathrm{c}}$ remains close to 0 , our model is identical to the original pom-pom model.

2.1.2. Backbone Orientation. The branch point friction constant $\xi_{\mathrm{b}}$ is defined as

$$
\xi_{\mathrm{b}}=2 \mathrm{kT} \frac{\tau_{\mathrm{a}}\left(\mathrm{s}_{\mathrm{c}}\right)}{\mathrm{a}_{\mathrm{eff}}^{2}} \mathrm{q}
$$

where $a_{\text {eff }}$ is the effective tube diameter and $\tau_{\mathrm{a}}\left(\mathrm{s}_{\mathrm{c}}\right)$ the relaxation time of the arms outside the tube. The entanglement molecular weight is assumed to be proportional to the reciprocal of the concentration of entangled strands, which is $\phi_{\mathrm{b}}$ in the case where no arms retraction takes place. Under strong flows, branch point withdrawal occurs which increases the length of the backbone tube. Actually, q retracted arm parts sc define along the backbone chain a single tube of length $\mathrm{s}_{\mathrm{b}}+2 \mathrm{~s}_{\mathrm{c}}$, reminding that tube concentration is defined as the ratio of the tube length to the total length of 
material. The concentration of entangled strands becomes therefore

$$
\frac{s_{b}+2 s_{c}}{2 q s_{a}+s_{b}}
$$

In that way the dilated tube primitive path $\phi_{\mathrm{b}} \mathrm{S}_{\mathrm{b}}$ becomes

$$
\frac{s_{b}+2 s_{c}}{2 q s_{a}+s_{b}} \cdot\left(s_{b}+2 s_{c}\right)
$$

Thus, the final equation for the backbone relaxation time is

$$
\tau_{b}\left(s_{c}\right)=\frac{4}{\pi^{2}}\left(s_{b}+2 s_{c}\right)^{2}\left(\phi_{b}+\frac{2 s_{c}}{2 q s_{a}+s_{b}}\right) \tau_{a}\left(s_{c}\right) q
$$

The orientation dynamics is described by the following differential equation using the tensor $\mathbf{S}$

$$
\frac{\partial \mathbf{S}}{\partial \mathrm{t}}=\mathbf{K} \cdot \mathbf{S}+\mathbf{S} \cdot \mathbf{K}^{\top}-2(\mathbf{S}: \mathbf{K}) \mathbf{S}-\frac{1}{\tau_{\mathrm{b}}}\left(\mathbf{S}-\frac{1}{3} \mathbf{I}\right)
$$

2.1.3. Backbone Stretch. As we consider that only the backbone segment is stretched, the equilibrium separation between branch points is still $\mathrm{sba}_{\text {eff }}$ and the effective elastic constant $\kappa=\mathrm{kT} / \mathrm{s}_{\mathrm{b}} \mathrm{a}^{2}$. Therefore, no effect of branch point withdrawal has to be taken into account on the stretch relaxation time

$$
\tau_{\mathrm{s}}=\mathrm{s}_{\mathrm{b}} \tau_{\mathrm{a}}(0) \mathrm{q}
$$

The stretch parameter $\lambda$ dynamics is described by the following differential equation:

$$
\frac{\partial \lambda}{\partial \mathrm{t}}=\lambda(\mathbf{K}: \mathbf{S})-\frac{1}{\tau_{\mathrm{s}}}(\lambda-1)
$$

2.1.4. Arm Retraction. The relaxation time of arm retraction is defined as the relaxation time of a segment of length $\mathrm{S}_{\mathrm{a}}-\mathrm{S}_{\mathrm{c}}$, which is $\tau_{\mathrm{a}}\left(\mathrm{S}_{\mathrm{c}}\right)$. Therefore, the differential equation describing the retraction dynamics remains

$$
\frac{\partial \mathrm{s}_{\mathrm{c}}}{\partial \mathrm{t}}=\left(\mathrm{q} \frac{\mathrm{s}_{\mathrm{b}}}{2}+\mathrm{s}_{\mathrm{c}}\right) \mathbf{K}: \mathbf{S}-\frac{1}{2 \tau_{\mathrm{a}}\left(\mathrm{s}_{\mathrm{c}}\right)}
$$

2.1.5. Expression for the Stress. For strain rates between $\tau_{\mathrm{b}}{ }^{-1}$ and $\tau_{\mathrm{a}}\left(\mathrm{S}_{\mathrm{c}}\right)^{-1}$ the main contribution to the stress becomes from the backbone. The "pom" arms are relaxed and are seen as a swelling purely viscous solvent. Reminding that for strong flows $\mathrm{s}_{\mathrm{c}}$ contributes to backbone orientation but not to backbone stretching, McL eish and Larson dissociate the stress into two parts. To be consistent, we must take into account in the definition of the stress that the concentration of topological obstacles increases when branch point withdrawal occurs. We therefore derive the foll owing equation for the stress tensor

$$
\boldsymbol{\sigma}=3 \mathrm{G}_{0}\left(\phi_{\mathrm{b}}+\frac{2 \mathrm{~s}_{\mathrm{c}}}{2 \mathrm{q} \mathrm{s}_{\mathrm{a}}+\mathrm{s}_{\mathrm{b}}}\right)\left(\phi_{\mathrm{b}} \lambda^{2}+\frac{2 \mathrm{qs}_{\mathrm{c}}}{2 \mathrm{q} \mathrm{s}_{\mathrm{a}}+\mathrm{s}_{\mathrm{b}}}\right) \mathbf{S}
$$

where the first coefficient $\phi_{b}+2 s_{d} /\left(2 q s_{a}+s_{b}\right)$ takes into account the dependence of the tube diameter on concentration of topological obstacles as discussed in section 2.1.2. Here again we recall that the dilated tube diameter is proportional to the reciprocal of tube concentration, which is $2 \mathrm{qs}_{d}\left(2 \mathrm{qs}_{\mathrm{a}}+\mathrm{s}_{\mathrm{b}}\right)+\phi_{\mathrm{b}}$. $\phi_{\mathrm{b}}$ accounts for stretched segments concentration and $2 q s_{d}\left(2 q s_{a}+\right.$ $\mathrm{S}_{\mathrm{b}}$ ) the volume fraction of withdrawn arm segments that contribute to orientation.

2.2. Global Set of Differential Equations and Relaxation Times. a. Dynamics of orientation tensor $\mathbf{S}$

$$
\frac{\partial \mathbf{S}}{\partial \mathrm{t}}=\mathbf{K} \cdot \mathbf{S}+\mathbf{S} \cdot \mathbf{K}^{\top}-2(\mathbf{S}: \mathbf{K}) \mathbf{S}-\frac{1}{\tau_{\mathrm{b}}}\left(\mathbf{S}-\frac{1}{3} \mathbf{I}\right)
$$

arm relaxation time

$$
\begin{aligned}
\tau_{\mathrm{a}}\left(\mathrm{s}_{\mathrm{c}}\right)=\tau\left(\mathrm{s}_{\mathrm{c}}\right) \exp \left[\frac { 1 5 } { 4 } \mathrm { S } _ { \mathrm { a } } \left(\frac{\left(1-\frac{\mathrm{s}_{\mathrm{c}}}{\mathrm{S}_{\mathrm{a}}}\right)^{2}}{2}-\right.\right. & \\
& \left.\left.\left(1-\phi_{\mathrm{b}}-\phi_{\mathrm{c}}\right) \frac{\left.\left(1-\frac{\mathrm{s}_{\mathrm{c}}}{\mathrm{S}_{\mathrm{a}}}\right)^{3}\right)}{3}\right)\right]
\end{aligned}
$$

where

$$
\tau\left(\mathrm{S}_{\mathrm{c}}\right)=\tau_{0}\left(\mathrm{~S}_{\mathrm{a}}-\mathrm{S}_{\mathrm{c}}\right)^{2} \sqrt{\frac{\pi}{v\left(\mathrm{~S}_{\mathrm{a}}-\mathrm{S}_{\mathrm{c}}\right)}}
$$

and

$$
\phi_{\mathrm{c}}=\frac{2 q s_{\mathrm{c}}}{2 q s_{\mathrm{a}}+\mathrm{s}_{\mathrm{b}}}
$$

backbone relaxation time

$$
\tau_{b}\left(s_{c}\right)=\frac{4}{\pi^{2}}\left(s_{b}+2 s_{c}\right)^{2}\left(\phi_{b}+\frac{2 s_{c}}{2 q s_{a}+s_{b}}\right) \tau_{a}\left(s_{c}\right) q
$$

b. Dynamics of stretch parameter $\lambda$

$$
\frac{\partial \lambda}{\partial \mathrm{t}}=\lambda(\mathbf{K}: \mathbf{S})-\frac{1}{\tau_{\mathrm{s}}}(\lambda-1)
$$

stretch relaxation time

$$
\tau_{\mathrm{s}}=\mathrm{s}_{\mathrm{b}} \tau_{\mathrm{a}}(0) \mathrm{q}
$$

c. Dynamics of arm withdrawal

$$
\frac{\partial s_{c}}{\partial t}=\left(q \frac{s_{b}}{2}+s_{c}\right) \mathbf{K}: \mathbf{S}-\frac{1}{2 \tau_{a}\left(s_{c}\right)}
$$

d. Expression for the stress

$$
\boldsymbol{\sigma}=3 \mathrm{G}_{0}\left(\phi_{\mathrm{b}}+\frac{2 \mathrm{~s}_{\mathrm{c}}}{2 \mathrm{q} \mathrm{s}_{\mathrm{a}}+\mathrm{s}_{\mathrm{b}}}\right)\left(\phi_{\mathrm{b}} \lambda^{2}+\frac{2 \mathrm{q} \mathrm{s}_{\mathrm{c}}}{2 \mathrm{qs} \mathrm{s}_{\mathrm{a}}+\mathrm{s}_{\mathrm{b}}}\right) \mathbf{S}
$$

2.3. Results. From our set of equations we can first notice that under slow flow conditions such that branch point withdrawal can be neglected $\left(\mathrm{s}_{\mathrm{c}}=0\right)$ our model is identical to the original pom-pom model. Nevertheless, under stronger flows, the model exhibits a singular behavior in both simple shear and uniaxial el ongation. The most interesting feature is its consequence on the relaxation time scale. As we can observe in eq 3 , when $\mathrm{s}_{\mathrm{c}}$ increases, two competing effects control the value of $\tau_{\text {a. }}$. The first one is the dynamic dilution effect that 


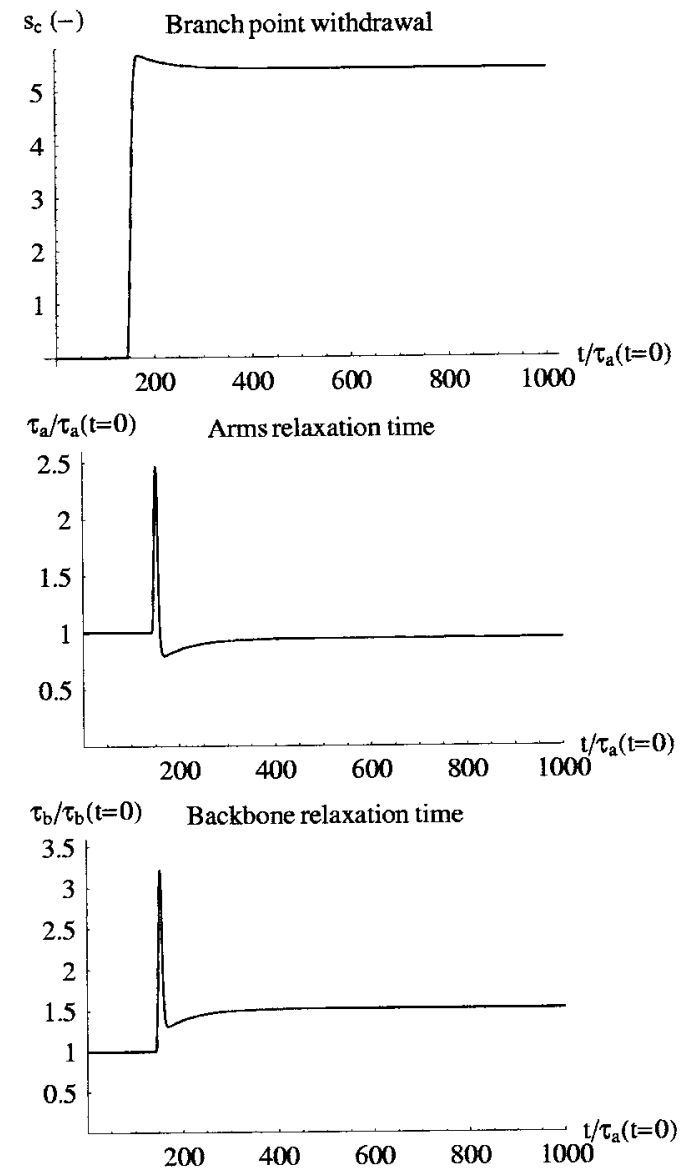

Figure 1. Shear modification of a pom-pom polymer.

decreases when arm retraction occurs, so that arm relaxation slows down. Thus, $\tau_{\mathrm{a}}$ increases. The other one is the branch length reduction which induces a decrease of $\tau_{\mathrm{a}}$. Actually, we must point out here that in some cases the Weissenberg number (with respect to arm dynamics) can be larger than unity depending on the higher transient value reached by $\tau_{\mathrm{a}}$ before it decreases. In this case one would have to include arms contribution to the stress. This will be the topic of a later work. Those small modifications of the initial pom-pom model without any further assumptions introduce naturally the effect of flow strength on the rheological properties of the material. As one will see in the following part, the behavior of the modified (i.e., processed) material can differ significantly from that of the native one.

2.3.1. Shear Modification Mechanism. Figure 1 shows the mechanism of "shear modification" for a pompom molecule with $s_{a}=30, s_{b}=30$, and $q=5$ under simple shear. The horizontal axis represents $t / \tau_{\mathrm{a}}(\mathrm{t}=0)$, the dimensionless time with respect to the initial value of $\tau_{\mathrm{a}}$. For $\tau_{\mathrm{a}}$ and $\tau_{\mathrm{b}}$, results are expressed in terms of the ratio of the instantaneous relaxation times and their initial value $\tau_{\mathrm{a}, \mathrm{b}}(\mathrm{t}) / \tau_{\mathrm{a}, \mathrm{b}}(\mathrm{t}=0)$. We adjusted the strength of the flow to $\mathrm{We}_{\mathrm{a}}=0.1$ in order to respect the initial pom-pom assumption of $\mathrm{We}_{a}<1$ (where $\mathrm{We}_{\mathrm{a}}$ is the Weissenberg number with respect to the arm relaxation time $\tau_{\mathrm{a}}$ ). On the contrary, the flow must be strong enough to induce chain stretching, $\mathrm{We}_{5}>1$ (where Wes is the Weissenberg number with respect to backbone stretching relaxation time $\tau_{\mathrm{s}}$ ). In such flow conditions, $\lambda$ increases until it reaches its maximal value of $q$, and then arm retraction takes place, reaching a plateau value close to 5 . As we can see, arm retraction induces
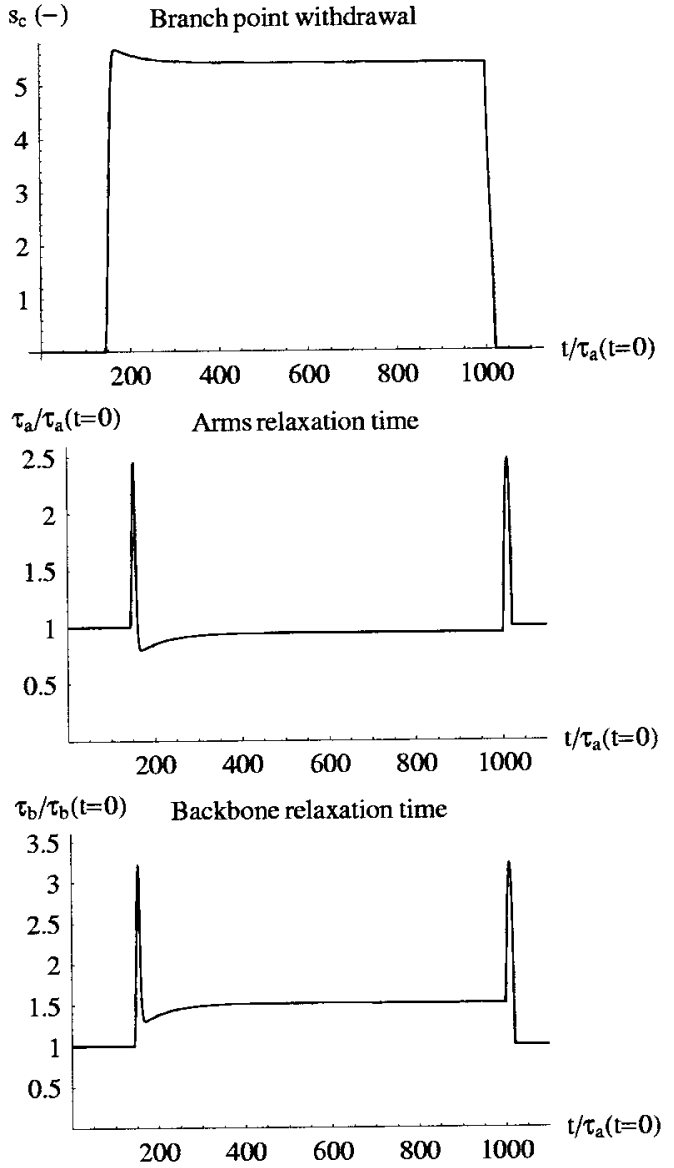

Figure 2. Shear modification recovery; flow is stopped at $\mathrm{t} / \tau_{\mathrm{a}}(\mathrm{t}=0)=1000$.

a peak of $\tau_{\text {a }}$ followed by a plateau value below the initial value.

2.3.2. Shear Modification Recovery. A key feature of shear modification phenomena is the anneal ing time. We already said that this time was described to be longer than the arm relxation time of the native polymer. This can be well understood considering that $\tau_{\mathrm{a}}$ and $\tau_{\mathrm{b}}$ go through a maximum during the shearing process. To recover its initial properties, the material has to take the same path. This means that the recovery of the material is much longer than the arm relaxation time of the annealed material because $\tau_{\mathrm{a}}$ and $\tau_{\mathrm{b}}$ can reach respectively 2.5 and 3 times the equilibrium values. Figure 2 shows the shear modification process up to $\mathrm{t} / \tau_{\mathrm{a}}(\mathrm{t}=0)=1000$ under simple shear. At that time the flow is stopped, and one can see the recovery phenomenon until the material reaches its initial properties at a time close to $\mathrm{t} / \tau_{\mathrm{a}}(\mathrm{t}=0)=1020$. Therefore, the time the melt needs to anneal is 20 times the arm relaxation time.

2.3.3. Rheological Properties. a. Behavior under Simple Shear. To investigatetherheological properties of a modified material and to compare results, we simulate some experiments from the literature. What has been done by many author s,9,11,15 is to shear modify a material using an extruder or a mixing device and to collect a sample and remold some tablets in order to make some rheological measurements. Authors are confident that the sample collection and preparation were fast enough to avoid recovery. We therefore assume that the global orientation of the molecul es is lost, but the microscopic topological modifications still sur- 


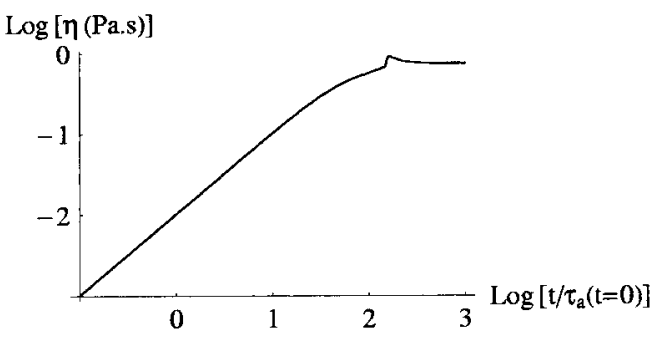

Figure 3. Transient shear viscosity before preshearing.

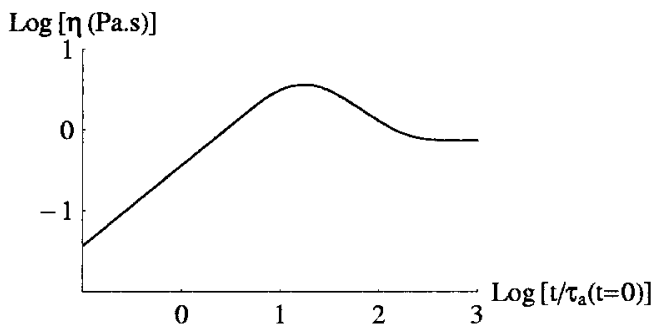

Figure 4. Transient shear viscosity after preshearing.

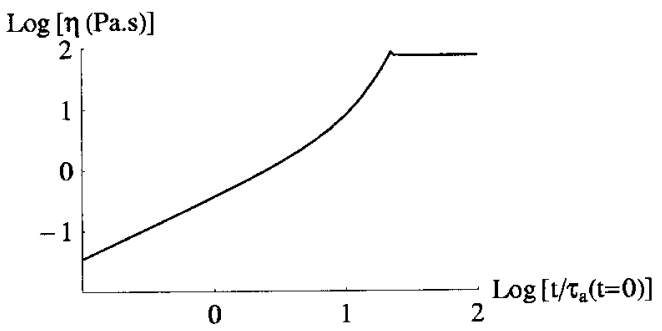

Figure 5. Uniaxial viscosity of the native polymer.

vive. In a first step we characterize its rheological behavior under shear (Figure 3). According to experimental conditions, we assume that an overall isotropic state is reached during sample preparation, but we consider that arm retraction and backbone stretching still survive according to the slow recovery kinetics. Then one has to enter the backbone stretching and branch point withdrawal values of the sheared material to initiate the calculation. We apply the same shear rate as during preshearing in order to make relevant comparisons with the native polymer. As $\tau_{\mathrm{a}}$ has a lower value, $\mathrm{We}_{a}$ is still lower than unity, and the basic pompom model assumptions are still respected.

The viscosity curve of the native material shows a singularity (Figure 3), corresponding to the dynamics of $\mathrm{S}_{\mathrm{c}}$ whereas the modified material has a smooth behavior with an overshoot of the viscosity (Figure 4). As the backbone chains remain stretched, no further contribution of the stretch parameter $\lambda$ on the evolution of the viscosity can occur. The behavior of the modified material is therefore radically different from that of the native one. Furthermore, as the relaxation times are lower, the dynamics of the backbone orientation and branch point withdrawal is different, too. It is now obvious that the level of arm retraction during the preshearing process controls the shear behavior of the flow modified material.

b. Uniaxial Elongation. The native material is highly branched, and as expected for strain rates higher than $\tau_{\mathrm{s}}{ }^{-1}$ (but still lower than $\tau_{\mathrm{a}}{ }^{-1}$ ), it shows the strain hardening behavior in uniaxial elongation (Figure 5).

The modified material exhibits a very different behavior under uniaxial el ongation. Actually, for thestrain rate value considered here (the same as for the native

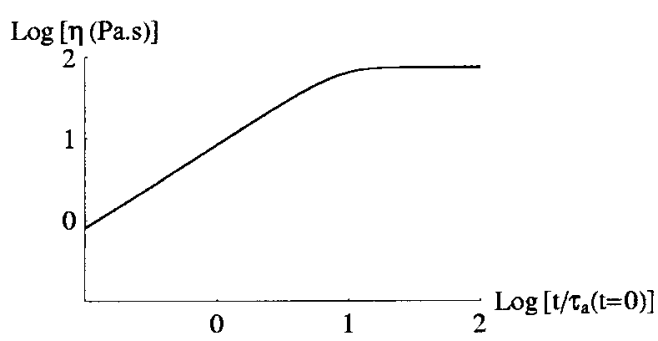

Figure 6. Uniaxial viscosity of the modified polymer.

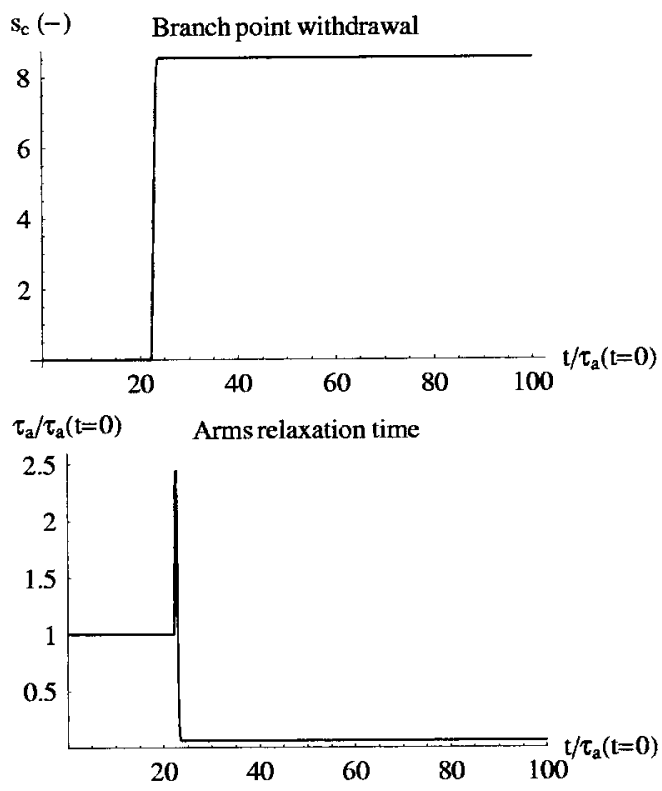

$\tau_{\mathrm{b}} / \tau_{\mathrm{b}}(\mathrm{t}=\mathbf{0}) \quad$ Backbone relaxation time

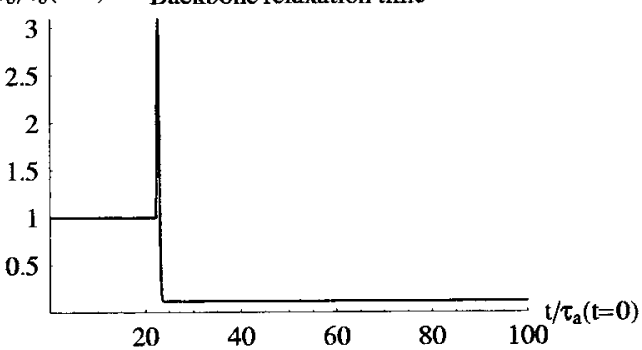

Figure 7. Elongation modification.

polymer), the melt shows no strain hardening behavior. As for shear flow, we can argue that if backbone chains remain stretched, no further stretch evolution can occur, annealing the strain hardening property (Figure 6). Furthermore, one can notice that rheological properties are more modified by el ongation than by simple shear. In fact, with the same strain rate, "elongation modification" (Figure 7) achieves higher branch point withdrawal ( $\mathrm{S}_{\mathrm{c}}$ is about 8 instead of 5 for shear) which induces lower values of the relaxation times than shear modification does (Figure 1), which is in good agreement with the previous work of Leblans and Bastiaansen. ${ }^{15}$

2.3.4. Effects of Topology. Having designed a molecular model to explain the origin of the shear or elongation modification mechanism, it is interesting to study now the effect of molecular topology. In this analysis, we make the dimensionless arm length $s_{a}$ vary from 3 to 90, keeping the same number of arms $(q=5)$ and the same backbone length $\left(s_{b}=30\right)$.

Samples are subjected to simple shear with a Weissenberg number relative to arm relaxation time $\mathrm{We}_{a}=$ 0.1 , from $t=0$ to $t=1000 \tau_{a}$. At that time, the flow is 

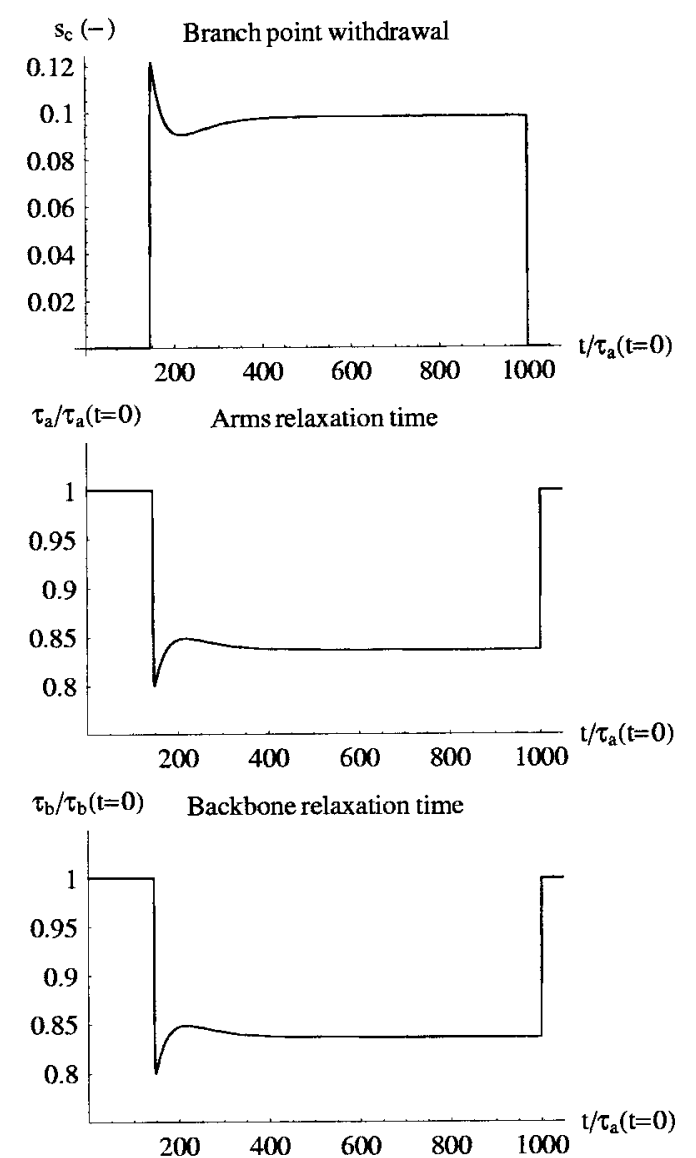

Figure 8. Recovery kinetics for $a s_{a}=3, s_{b}=30, q=5$ pompom molecule; flow is stopped at $\mathrm{t} / \tau_{\mathrm{a}}(\mathrm{t}=0)=1000$.

stopped, and the melt begins to recover its initial properties. For molecules with short-chain branches ( $s_{a}$ $=3$ ), we can notice in Figure 8 that branch point withdrawal reaches a low equilibrium value of 0.1 , which means that the volume fraction of retracted arm material $\phi_{c}$ remains negligible compared to the vol ume fraction of material inside a tube $\phi_{\mathrm{b}}$. Ther efore, in eq 3 , only the branch length reduction $\mathrm{s}_{\mathrm{a}}$ control $\mathrm{s}$ the evolution of $\tau_{\mathrm{a}}$ and $\tau_{\mathrm{b}}$. Finally, the relaxation times reach a value lower by $15 \%$. For material with very long-chain branches $\left(s_{a}=90\right)$, subjected to the same simple shear flow strength $\left(\mathrm{We}_{\mathrm{a}}=0.1\right)$, branch point withdrawal reaches a higher value close to 23 , which induces a significant increase of $\phi_{c}$. $\phi_{c}$ becomes nonnegligible compared to $\phi_{\mathrm{b}}$. In eq 3 both arm length reduction and dynamic dilution control the evolution of $\tau_{\text {a }}$. Therefore, the relaxation times increase in a first step by a factor of 200 for the arms and by a factor of 550 for the backbone. In this case, the recovery mechanism is very slow $\left(3500 \tau_{\text {a }}(t=0)\right)$.

In processing conditions, these very long-chain branched molecules are more affected by shear modification, and their recovery kinetics is so slow that the melt properties remain very different from the native state during the whole processing time. This is in qualitative agreement with the experimental results of Ritzau et al. ${ }^{14}$

\section{Discussion}

Starting from these results, we can discuss the qualitative agreement between the shear modification effects derived from the molecular mechanism described by this model and the experimental evidence. First we
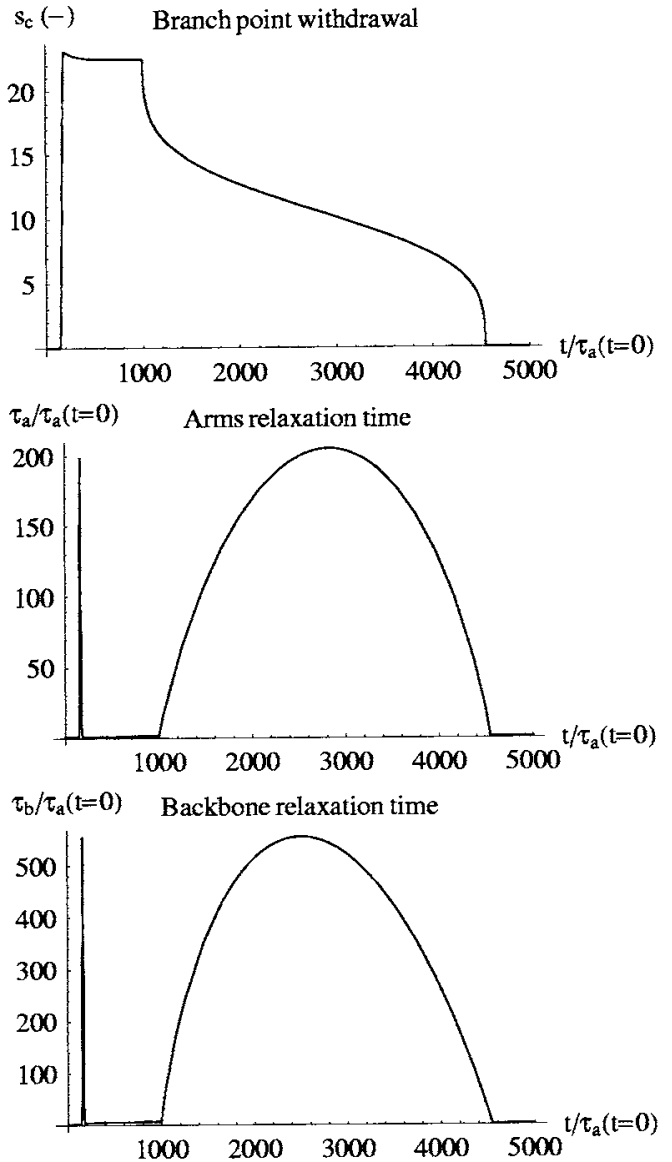

Figure 9. Recovery kinetics for $a s_{a}=90, s_{b}=30, q=5$ pompom molecule; flow is stopped at $\mathrm{t} / \tau_{\mathrm{a}}(\mathrm{t}=0)=1000$.

must point out that only long-chain branched molecules are concerned by this phenomenon. For short-chain branched molecules, the modification is minor whereas long-chain branched molecules properties are strongly affected. This is in qualitative agreement with experiments, for which it was reported that material with broader molecular weight distribution and long-chain branching (such as LDPE from autoclave synthesis) are more sensible. We believe that those materials are more affected because they contain much more long-chain branched molecules compared to the other materials. Relaxation times of the modified material are lower, resulting in a smaller intrinsic elasticity and no strain hardening behavior. Another important experimental observation is the annealing time needed to reverse the modifications which is longer than expected. We can now say that the side chain branches alignment hypothesis is not in contradiction with the reptation theory, and it is now acknowl edged that the annealing time is longer than the arm relaxation time of the native polymer because of the dynamic dilution effect which increases the global time scale of the material as side chain alignment occurs. Finally, as was assumed by the same authors, "el ongation modification" is more effective than "shear modification" despite too few experimental results were reported in the literature.

Our approach has some similarities with the work of Blackwell et al. ${ }^{21}$ in the sense that we al so consider the effect of branch point withdrawal on the time scale of a pom-pom molecule. These authors investigated the effect of a coupling mechanism between a local branch point withdrawal and the backbone stretch. Their main conclusion is that the relaxation time for the stretch is 
reduced improving the fit to transient and steady-state data. Furthermore, this avoids the unphysical cusp in extensional viscosity caused by the sharp transition from the stretch dynamics to the branch point withdrawal dynamics when maximal backbone stretch is reached. Nevertheless, there are major differences between this approach and ours. We investigate some much larger branch points displacements which induce some significant changes in the entanglement network and therefore some modifications on all relaxation times.

What is described here is a global change of the "apparent topology" of the molecule from an initial state where branched molecules entangle each other to a state where side chain branches and backbone are aligned in a same linear "supertube". This new "apparent topology" induces a strong modification of rheological properties for long-chain branched molecules. On an experimental point of view, reported results show less drastic modifications, and we believe that polydispersity may smooth topological changes because only long-chain branched molecules undergo branch point withdrawal.

We must also discuss the increase of the plateau modulus values predicted by our model. On eq 12 one can argue that the shear modulus increases with $\mathrm{s}_{c}$ which is in qualitative disagreement with the intuitive idea that disentanglement increases with arm retraction and with deformation. Nevertheless, in the original pom-pom model, arms do not contribute to the effective entanglement network for flow rates below $\tau_{\mathrm{a}}{ }^{-1}$. Therefore, the decrease in arms length does not induce any change in the molecular weight between entanglements $\mathrm{M}_{\mathrm{e}}$. On the contrary, the increase of backbone length makes $\mathrm{M}_{e}$ decrease and therefore the plateau modulus increase. Taking into account arms to the entanglement molecular weight is thus an important issue which will be treated in a future work.

\section{Conclusion}

The aim of this work was to use a theoretical model based on a recent molecular dynamics picture of LCB polymers to discuss the origin of the phenomenon of "shear modification". Small changes in the original pompom model introduce naturally a dependence of rheological properties on flow history. Side chain branches partly align each other within a single backbone tube. This change in apparent topology leads to an evolution of the global morphology which induces a very significant modification of relaxation times. This modification has a great effect on the recovery kinetics of the initial properties. The annealing time may be larger by several orders of magnitude compared with the longest relaxation time of the native material. This result explains why there is no contradiction between the side chain alignement hypothesis proposed by Leblans and Bastiaansen ${ }^{15}$ and the reptation theory. As previously discussed by these authors, our results show that "elongation modification" is more effective than "shear modification" for the same flow strength. F urthermore, the effect of molecular topology is important, and results demonstrate that very long-chain branched molecules are highly affected by "shear modification", whereas short-chain branched molecules (linear polymer) undergo very small changes.

All our results are in qualitative agreement with previously reported experimental results. However, we have only studied flow conditions respecting an upper limit of strain rates such that $\mathrm{We}_{a}<1$. This is an important restriction from an experimental point of view, especially for very long-chain branched molecules. We therefore want to include the arms contribution to the stress in order to study stronger flow conditions and try to achieve a quantitative comparison with experimental results. This will be the aim of our future work. Eventually, it will be of highest importance from an industrial point of view to study an effective way to control this "flow modification" in order to get a powerful control of material properties during the process and to achieve higher processability and performances.

\section{References and Notes}

(1) Hanson, D. E. Polym. Eng. Sci. 1969, 9, 405.

(2) Prichard, J . H.; Wissbrun, K. F. J . Appl. Polym. Sci. 1969, $13,233$.

(3) Rokudai, M. J . Appl. Polym. Sci. 1979, 23, 463.

(4) Rokudai, M.; Mihara, S.; Fujiki, T. J . Appl. Polym. Sci. 1979, 23, 3289.

(5) Rokudai, M.; Fujiki, T. J . Appl. Polym. Sci. 1981, 26, 1343.

(6) Maxwell, B.; Breckwoldt, A. J . Rheol. 1981, 25, 55.

(7) Rudin, A. Polym. Eng. Sci. 1983, 23, 422.

(8) Roovers, J. Macromolecules 1984, 17, 1196.

(9) Teh, J. W.; Rudin, A.; Schreiber, H. P. Polym. Eng. Sci. 1985, 30, 1345 .

(10) Ram, A.; Izailov, L. J . Appl. Polym. Sci. 1986, 31, 85.

(11) Ritzau, G. Int. Polym. Process. 1987, 1, 188.

(12) McLeish, T. C. B. Macromol ecules 1988, 21, 1062.

(13) Ball, R. C.; McLeish, T. C. B. Macromolecules 1989, 22, 1911.

(14) Ritzau, G. Polym. Eng. Sci. 1989, 29, 214.

(15) Leblans, P. J . R.; Bastiaansen, C. Macromolecules 1989, 22, 3312.

(16) Van Prooyen, M.; Bremner, T.; Rudin, A. Polym. Eng. Sci. 1994, 34, 570

(17) Van Prooyen, M.; Peemoeller, H.; Rudin, A. Polym. Eng. Sci. 1996, 36, 2001

(18) McLeish, T. C. B.; Larson, R. G. J . Rheol. 1998, 42, 81

(19) Kappès, N. Ph.D. Thesis, Université de Pau et des Pays de I'Adour, 1998.

(20) McLeish, T. C. B.; Allgaier, J .; Bick, D. K.; Bishko, G.; Biswas, P.; Blackwell, R.; Blottiere, B.; Clarke, N.; Gibbs, B.; Groves, D. J .; Hakiki, A.; Heenan, R. K.; J ohnson, J . M.; Kant, R.; Read, D. J .; Young, R. N. Macromolecules 1999, 32, 6734.

(21) Blackwell, R. J .; McLeish, T. C. B.; Harlen, O. G. J . Rheol. 2000, 44, 121

MA021195T 